\title{
Pretreatment Lesional Volume Impacts Clinical Outcome and Thrombectomy Efficacy
}

\author{
Yu Xie, MD, MSc, ${ }^{1}$ Catherine Oppenheim, MD, $\mathrm{PhD}_{1}{ }^{2}$ \\ Francis Guillemin, MD, PhD, ${ }^{3}$ Vincent Gautheron, $\mathrm{MD}^{2}$ \\ Benjamin Gory, MD, PhD $\oplus^{4}{ }^{4}$ Hélène Raoult, MD, PhD, ${ }^{5}$ \\ Sébastien Soize, MD, PhD, ${ }^{6}$ Jacques Felblinger, $\mathrm{PhD}_{1}^{1,7}$ \\ Gabriela Hossu, PhD, ${ }^{7}$ and Serge Bracard, MD (1) ${ }^{1,8}$; \\ on behalf of the THRACE investigators
}

\begin{abstract}
Objective: We aimed to characterize the association between pretreatment lesional volume measured on diffusionweighted images and functional outcome, and estimate the impact on thrombectomy efficacy for ischemic stroke with anterior proximal intracranial arterial occlusion.

Methods: Anterior circulation ischemic stroke patients who had pretreatment diffusion-weighted imaging in the THRACE study were included. Lesional volume was semiautomatically segmented. Logistic regression was applied to model clinical outcome as a function of lesional volume. Outcomes included functional independence (modified Rankin Scale [mRS] 0-2), degree of disability (ordinal mRS 0-6), and mortality at 3 months.

Results: Of 298 included patients, with median lesional volume 17.2ml (interquartile range [IQR], 9.2-51.8) and median mRS 2 (IQR, 1-4), 51.0\% achieved functional independence. Increased lesional volume was an independent predictor for a lower probability of functional independence (odds ratio [OR], 0.90 [95\% confidence interval $\{\mathrm{Cl}\}$, 0.81-0.99] per 10ml; $p<0.001$ ), a less favorable degree of disability (common OR, 0.86 [95\% Cl, 0.81-0.90] per $10 \mathrm{ml} ; p<0.001)$, and a higher mortality rate $(\mathrm{OR}, 1.21[95 \% \mathrm{Cl}, 1.08-1.37]$ per $10 \mathrm{ml} ; p<0.001)$. For additional thrombectomy, the number of patients needed to treat to achieve functional independence in 1 patient increased with lesional volume ( 10 for a volume of $80 \mathrm{ml} ; 15$ for $135 \mathrm{ml}$ ). No significant treatment-by-dichotomized volume interaction for functional independence and mortality was observed.

Interpretation: Pretreatment lesional volume is an independent predictor for functional outcome in acute ischemic stroke with proximal intracranial occlusion. The clinical benefit of adding mechanical thrombectomy to thrombolysis decreased with the increase of lesional volume.
\end{abstract}

ANN NEUROL 2018;83:178-185

Pecent randomized clinical trials have demonstrated The clinical benefit of mechanical thrombectomy (MT) in acute ischemic stroke patients with anterior circulation proximal intracranial occlusions. ${ }^{1-8}$ In the majority of these trials, patients were evaluated with computed tomography (CT) and selected by

View this article online at wileyonlinelibrary.com. DOI: 10.1002/ana.25133

Received Oct 16, 2017, and in revised form Dec 28, 2017. Accepted for publication Dec 29, 2017.

Corresponding Author: Pr Serge Bracard, Department of Diagnostic and Interventional Neuroradiology, University Hospital of Nancy, 54035 Nancy, France. E-mail: s.bracard@chru-nancy.fr

From the ${ }^{1}$ IADI, U1254, INSERM, Université de Lorraine, CHRU Nancy, Nancy, France; ${ }^{2}$ Department of Neuroradiology, Sainte-Anne Hospital and ParisDescartes University, INSERM U894, Paris, France; ${ }^{3} \mathrm{CIC} 1433$ Epidémiologie Clinique, INSERM, Université de Lorraine, CHRU Nancy, Nancy, France; ${ }^{4}$ INSERM U1237, "Physiopathology and Imaging for Neurological Disorders (PhIND)", GIP Cyceron, Caen, France; ${ }^{5}$ Department of Neuroradiology, University Hospital of Rennes, Rennes, France; ${ }^{6}$ Department of Neuroradiology, University Hospital of Reims, Reims, France; ${ }^{7} \mathrm{CIC} 1433$ Innovation

Technologique, INSERM, Université de Lorraine, CHRU Nancy, Nancy, France; and ${ }^{8}$ Department of Diagnostic and Interventional Neuroradiology, CHRU Nancy, Nancy, France

Please see a supplementary file for the list of THRACE study investigators and their affiliations.

Additional supporting information can be found in the online version of this article. 


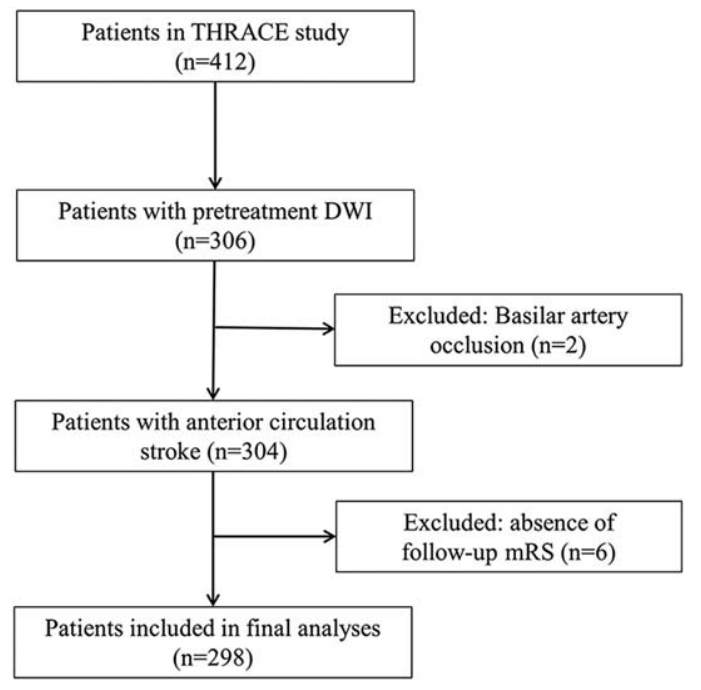

FIGURE 1: Patient selection flowchart. DWI= diffusionweighted imaging; $\mathrm{mRS}=$ modified Rankin Scale.

pretreatment ASPECTS (Alberta Stroke Program Early CT Score) threshold, and patients with large stroke volume were excluded. Infarct volume at follow-up was shown to be a prognostic biomarker ${ }^{9-11}$; however, uncertainty remained as to the optimal baseline imaging profile for favorable clinical outcome after MT. Extensive data from previous studies support diffusion-weighted imaging (DWI) as the most accurate imaging sequence available for estimating the ischemic core in acute ischemic stroke patients. ${ }^{12}$ To date, evidence with regard to the association between pretreatment lesional volume measured on DWI and functional outcome is relatively weak and is limited to retrospective single-center studies and thrombolysis therapy. ${ }^{13-16}$ Moreover, the effect of pretreatment lesional volume on the benefit of MT has yet to be determined.

The THRACE study ${ }^{6}$ provided a unique opportunity to evaluate the association between pretreatment lesional volume measured on DWI and both functional outcome and MT efficacy, because of the use of DWI in pretreatment imaging in a large proportion of patients and the absence of exclusion criteria on the basis of pretreatment lesional volume.

In this substudy, we aimed to characterize the relationship between pretreatment lesional volume measured on DWI and the functional outcome of ischemic stroke, and estimate the impact of pretreatment lesional volume on MT efficacy for acute ischemic stroke due to proximal intracranial arterial occlusion.

\section{Patients and Methods}

\section{Study Design and Participants}

The THRACE study (Clinical Trial Registration-URL: http:// www.clinicaltrials.gov; unique identifier: NCT01062698) was a randomized, controlled trial done in 26 centers in France, comparing intravenous thrombolysis plus MT (IVTMT) and intravenous thrombolysis alone (IVT) in patients with acute ischemic stroke attributed to proximal arterial occlusion, aged 18 to 80 years, and with an NIHSS (National Institutes of Health Stroke Scale) score of 10 to 25 . The study design and patient inclusion criteria have been described in detail previously. ${ }^{6}$ The study protocol was approved by the CPP (Comité de Protection des Personnes) Est III Ethics Committee and the research boards of the participating centers. All patients or their legal representatives provided written informed consent. In our THRACE substudy, we included anterior circulation stroke patients who had pretreatment DWI.

\section{Imaging and Clinical Assessment}

Pretreatment infarct lesions were semiautomatically segmented by Olea Sphere (Olea Medical SAS, La Ciotat, France) after applying a threshold of apparent diffusion coefficient (ADC) of $0.6 \times 10^{-3} \mathrm{~mm}^{2} / \mathrm{s}$ on ADC maps, ${ }^{17-19}$ which were calculated from DWI acquisitions. All the lesion masks were confirmed by two neuroradiologists and were manually corrected, if necessary.

Demographic and clinical data and functional outcome were extracted from the THRACE database. Primary outcome was the proportion of patients achieving functional independence at 3 months, defined by a score of 0 to 2 on the modified Rankin Scale (mRS; ranging from 0 [no symptoms] to 6 [death]). Secondary outcomes were (1) degree of disability at 3 months, assessed by the ordinal mRS from 0 to 6 with ranks 5 and 6 combined into a single category, and (2) mortality at 3 months. Cerebral reperfusion was assessed by angiography and reviewed by three experienced interventional neuroradiologists under a consensus evaluation for patients who received thrombectomy. Substantial reperfusion was defined as a modified Thrombolysis in Cerebral Infarction (mTICI) scale score of $2 b$ or 3 , corresponding to reperfusion of at least $50 \%$ of the affected vascular territory.

\section{Statistical Analysis}

Baseline characteristics and functional outcome were compared between patients in the IVT group and the IVTMT group. Non-Gaussian continuous variables are presented as median and interquartile range (IQR) while categorical variables are presented as proportions. Comparisons were performed by Wilcoxon test and chi-square test, respectively. $p$ values were twosided and $p=0.05$ was chosen as the significance level.

ASSOCIATION BETWEEN PRETREATMENT LESIONAL VOLUME AND FUNCTIONAL OUTCOME. Univariate and multivariate logistic analyses evaluated the association between lesional volume and clinical outcomes (binomial logistic regression for binarized mRS and ordinal logistic regression for ordinal mRS). Factors that had predictive value for clinical outcome in the main THRACE study ${ }^{6}$ (age, baseline NIHSS score, occlusion location, ASPECTS, blood glucose, and Fazekas score) were included as covariates in the present analysis. Variables with a $p$ value $<0.20$ in univariate analysis were included in the multivariate model. The final model was 
TABLE 1. Demographic and Clinical Baseline Characteristics of Patients

\begin{tabular}{|c|c|c|c|c|}
\hline & $\begin{array}{l}\text { All Patients } \\
(\mathrm{n}=304)\end{array}$ & $\begin{array}{l}\text { IVT Group } \\
(\mathbf{n}=155)\end{array}$ & $\begin{array}{l}\text { IVTMT Group } \\
(\mathrm{n}=149)\end{array}$ & $p$ \\
\hline Age, years & $67(54-74)$ & $68(54-75)$ & $67(54-74)$ & 0.64 \\
\hline Male sex & $159 / 304(52.3)$ & $72 / 155(46.5)$ & $87 / 149(58.4)$ & 0.05 \\
\hline Diabetes mellitus & $31 / 301(10.3)$ & $21 / 155(13.5)$ & $10 / 146(6.8)$ & 0.09 \\
\hline Hypertension & $155 / 301(51.5)$ & $86 / 155(55.5)$ & $69 / 146(47.3)$ & 0.19 \\
\hline Tobacco (current or past) & $117 / 274(42.7)$ & $56 / 142(39.4)$ & $61 / 132(46.2)$ & 0.31 \\
\hline Hypercholesterolemia & $142 / 271(52.4)$ & $80 / 142(56.3)$ & $62 / 129(48.1)$ & 0.21 \\
\hline Coronary disease & $42 / 290(14.5)$ & $22 / 148(14.9)$ & $20 / 142(14.1)$ & 0.98 \\
\hline History of stroke & $19 / 294(6.5)$ & $10 / 153(6.5)$ & $9 / 141(6.4)$ & 0.99 \\
\hline Admission blood glucose, g/l & $1.2(1.0-1.4)$ & $1.2(1.0-1.4)$ & $1.2(1.0-1.4)$ & 0.69 \\
\hline Admission NIHSS score & $18(14-20)$ & $17(14-21)$ & $18(14-20)$ & 0.83 \\
\hline $\begin{array}{l}\text { Time from stroke onset to } \\
\text { imaging, min }\end{array}$ & $114(89-139)$ & $113(92-140)$ & $114(86-139)$ & 0.54 \\
\hline $\begin{array}{l}\text { Time from stroke onset to } \\
\text { randomization, } \min \end{array}$ & $166(139-195)$ & $168(138-198)$ & $165(143-194)$ & 0.72 \\
\hline Occlusion location, ICA:M1 & $47: 257$ & $31: 124$ & $16: 133$ & $0.04^{*}$ \\
\hline ASPECT score & $7(5-9)$ & $7(5-9)$ & $7(6-8)$ & 0.89 \\
\hline Fazekas score & $1(0-1)$ & $1(0-2)$ & $1(0-1)$ & 0.11 \\
\hline Infarct laterality, left hemisphere & $145 / 304(47.7)$ & $69 / 155(44.5)$ & $76 / 149(51.0)$ & 0.31 \\
\hline \multicolumn{5}{|c|}{$\begin{array}{l}\text { Continuous variables are presented as median (interquartile range); categorical variables are presented as no. }(\%) . \\
{ }^{*} p<0.05 . \\
\text { IVT = intravenous thrombolysis; IVTMT = intravenous thrombolysis plus mechanical thrombectomy; NIHSS = National Institutes of Health } \\
\text { Stroke Scale; ICA = intracranial internal carotid artery; M1 = proximal portion of middle cerebral artery; ASPECT = Alberta Stroke Program Early } \\
\text { CT Score. }\end{array}$} \\
\hline
\end{tabular}

selected using Akaike's information criterion (AIC). Adjusted odds ratios (ORs) or common odds ratios (cORs) with 95\% confidence intervals (CIs) were calculated. The estimated probability of achieving functional independence according to age, baseline NIHSS score, and lesional volume for each patient was depicted by three-dimensional scatterplots. Fitted surfaces were added to describe the association between age, baseline NIHSS score, and lesional volume on the likelihood of functional independence. The reperfusion status was also included in the model in a per-protocol analysis of the IVTMT group (patients assigned to the IVTMT group who did not have thrombectomy were excluded from the analysis).

ASSOCIATION BETWEEN PRETREATMENT LESIONAL VOLUME AND THROMBECTOMY EFFICACY ON FUNCTIONAL OUTCOME. The association between pretreatment lesional volume and functional independence was evaluated in the IVTMT group and compared with the IVT group. The number needed to treat (NNT) ${ }^{20}$ was calculated to estimate the number of patients needed to treat to obtain 1 patient achieving functional independence after thrombectomy through the range of lesional volume. The interaction of treatment-by-dichotomized lesional volume for functional independence and mortality was assessed with two volume thresholds suggested in previous studies: 70 and $100 \mathrm{ml}^{15,21}$ All the statistical analyses were performed using statistical software R (version 3.3.2; R Foundation for Statistical Computing, Vienna, Austria).

\section{Results}

Of 412 patients in the THRACE study, 304 met the inclusion criteria (106 patients without pretreatment DWI examination and 2 with basilar artery occlusion were excluded; Fig 1), including 155 (51.0\%) patients in the IVT group and $149(49.0 \%)$ in the IVTMT group. The cohort was composed of $159(52.3 \%)$ men and had a median age of 67 (IQR, 54-74) years. Baseline characteristics of the included patients are presented in Table 1. No significant differences in baseline characteristics were 


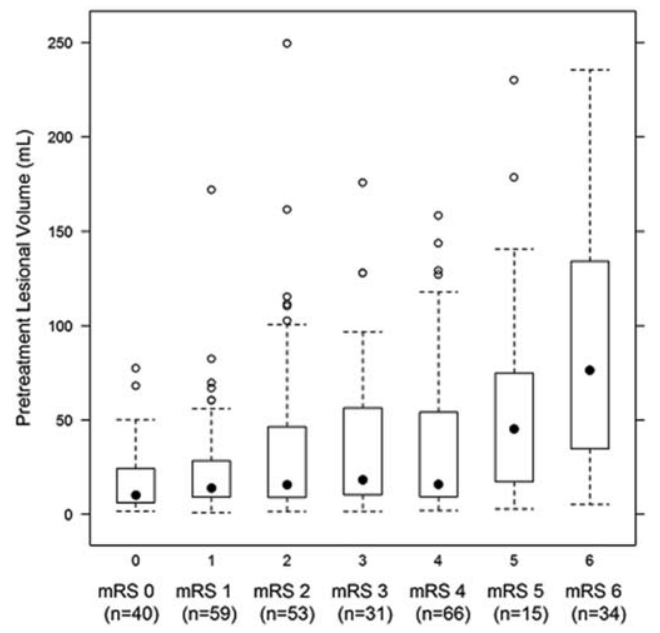

FIGURE 2: Box-and-whisker plot showing the distribution of the pretreatment lesional volume for each modified Rankin Scale (mRS) category. The boundaries of the box indicate the 25th and 75th percentiles and the black dots indicate the median values. The whiskers represent the lowest and highest values in the 25 th percentile minus 1.5 interquartile range (IQR) and 75th percentile plus $1.5 \mathrm{IQR}$, respectively. Circles indicate outliers.

observed between the two groups except for the occlusion location.

Furthermore, 6 patients without follow-up mRS evaluation were excluded, leaving 298 patients (IVTMT group, $\mathrm{n}=148$; IVT group, $\mathrm{n}=150$ ) for the subsequent analyses (median age, 67 years [IQR, 54-74]; women, $47.3 \%)$.

\section{Association Between Pretreatment Lesional Volume and Functional Outcome}

For the 298 patients, median pretreatment lesional volume was $17.2 \mathrm{ml}$ (IQR, 9.2-51.8) and median $\mathrm{mRS}$ was 2 (IQR, 1-4), with 152 (51.0\%) achieving functional independence at 3 months. The distribution of lesional volume for each mRS category is shown in Figure 2. Increased ordinal mRS was associated with a larger lesional volume (Spearman's $\rho$ correlation coefficient, 0.34; $p<0.0001$ ). Best regression models for the association between pretreatment lesional volume and functional outcomes and death are presented in Table 2. Lesional volume, together with age, baseline NIHSS score, intracranial occlusion location, and blood glucose level, were independent predictors for both functional independence and degree of disability. ASPECTS was not chosen to be part of the final regression model after the model selection using the AIC. The probability of achieving functional independence decreased with the increase of lesional volume (per 10ml: OR, 0.90; 95\% CI, $0.81-0.99 ; p<0.001)$. The degree of disability increased with the increase of lesional volume (per $10 \mathrm{ml}$ : cOR, 0.86; 95\% CI, 0.81-0.90; $p<0.001)$. The associations between lesional volume, age, baseline NIHSS score, and adjusted probability of functional independence were depicted by three-dimensional scatterplots with fitted surfaces (Fig 3). The mortality rate increased with the increase of lesional volume (per $10 \mathrm{ml}$ : OR, 1.21; 95\% CI, 1.08-1.37; $p<0.001$ ). When the reperfusion status was included as covariate in the per-protocol

\begin{tabular}{|c|c|c|c|c|c|c|}
\hline & $\begin{array}{l}\text { OR }(95 \% \mathrm{CI}) \\
\text { for Functional } \\
\text { Independence }\end{array}$ & $p$ & $\begin{array}{l}\text { cOR }(95 \% \text { CI }) \\
\text { for Degree } \\
\text { of Disability }\end{array}$ & $p$ & $\begin{array}{l}\text { OR }(95 \% \mathrm{CI}) \\
\text { for Mortality }\end{array}$ & $p$ \\
\hline Age & $0.96(0.93-0.98)$ & 0.001 & $0.97(0.95-0.99)$ & 0.001 & $1.06(1.02-1.11)$ & 0.002 \\
\hline Baseline NIHSS score & $0.89(0.83-0.95)$ & 0.001 & $0.91(0.86-0.96)$ & 0.001 & & \\
\hline Occlusion location (M1) & $3.03(1.31-7.52)$ & 0.011 & $2.04(1.12-3.72)$ & 0.020 & & \\
\hline Blood glucose level & $0.38(0.17-0.81)$ & 0.020 & $0.62(0.37-0.99)$ & 0.054 & & \\
\hline Fazekas score & $0.76(0.55-1.04)$ & 0.099 & $0.84(0.65-1.08)$ & 0.171 & & \\
\hline Lesional volume (per $10 \mathrm{ml}$ ) & $0.90(0.80-0.99)$ & $<0.001$ & $0.86(0.81-0.90)$ & $<0.001$ & $1.21(1.08-1.37)$ & $<0.001$ \\
\hline
\end{tabular}

Functional independence was defined by a modified Rankin Scale score 0 to 2 at 3 months. Degree of disability was assessed by ordinal modified Rankin Scale scores ranging from 0 to 6, with ranks 5 and 6 combined into a single category. Patients with missing values $(\mathrm{n}=12)$ were excluded from this analysis.

NIHSS = National Institutes of Health Stroke Scale; M1 = proximal portion of middle cerebral artery; OR = odds ratio; $c \mathrm{OR}=$ common odds ratio; $\mathrm{CI}=$ confidence interval. 

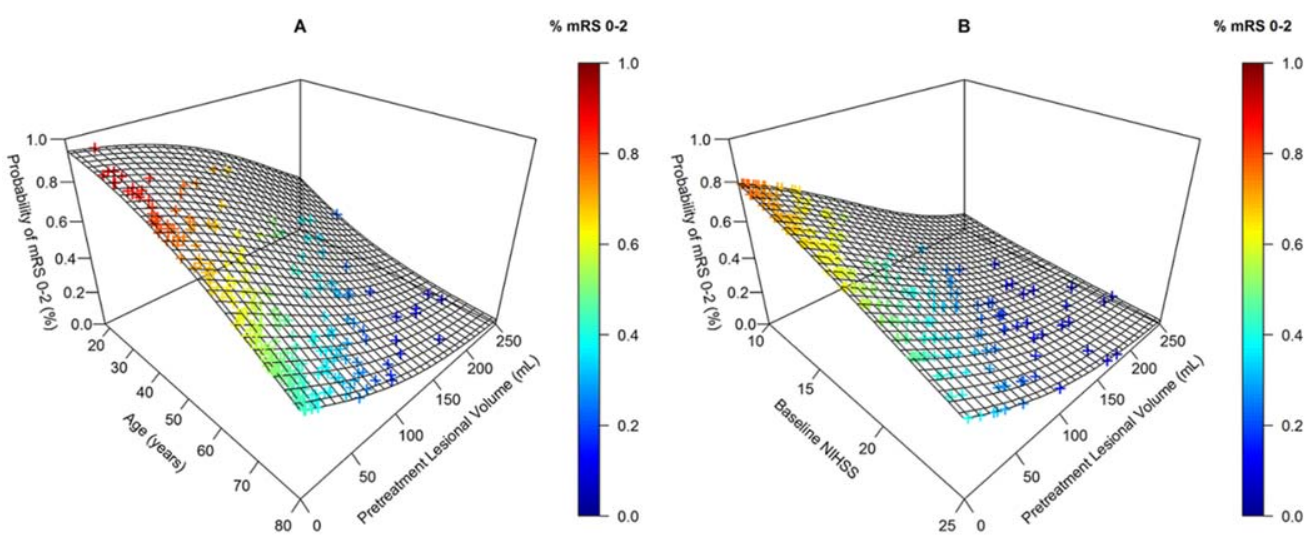

FIGURE 3: Three-dimensional scatterplots with fitted surfaces illustrating the association between age (A) or baseline NIHSS score (B), and pretreatment lesional volume, and adjusted probability of functional independence (mRS 0-2) at 3 months. The colored crosses indicate the estimated probability of functional independence for each patient in our cohort. $\mathrm{mRS}=$ modified Rankin Scale; NIHSS = National Institutes of Health Stroke Scale.

analysis $(\mathrm{n}=100)$, the increase of lesional volume was still associated with a decreased probability of functional independence (per 10ml: OR, 0.87; 95\% CI, 0.75-0.99; $p=0.05)$ and an increased degree of disability (per 10ml: cOR, 0.90; 95\% CI, 0.80-0.99; $p=0.04)$.

\section{Association Between Pretreatment Lesional Volume and Thrombectomy Efficacy on Functional Outcome}

The median pretreatment lesional volume was $18.4 \mathrm{ml}$ (IQR, 9.9-45.3) in the IVTMT group, and $15.5 \mathrm{ml}$ (IQR, 8.7-60.1) in the IVT group $(p=0.96)$. The median NIHSS score at 24 hours was 9 (IQR, 4-18) in the IVTMT group, and 12 (IQR, 6-19) in the IVT group $(p=0.08)$. The median mRS was 2 (IQR, $1-4)$ in the IVTMT group, and 3 (IQR, 1-4) in the IVT group $(p=0.21)$. Compared to IVT alone, IVTMT increased the proportion of patients achieving functional independence by $11.4 \%(95 \%$ CI $[-0.5 ; 23.4] ; p=0.06)$. In the IVTMT group, 46 (63.9\%) of 72 patients achieving substantial reperfusion and $7(25.0 \%)$ of 28 without substantial reperfusion had functional independence at 3 months. Substantial reperfusion increased the proportion of patients achieving functional independence by $38.9 \%$ (95\% CI $[16.9 ; 60.9] ; p=0.001)$. With the increase of pretreatment lesional volume, the probability of achieving functional independence decreased in both the IVTMT group and the IVT group (Fig 4). Adjusted OR for lesional volume per $10 \mathrm{~mL}$ was 0.87 (95\% CI, $0.77-$ $0.98 ; p=0.02)$ in the IVTMT group, $0.89(95 \% \mathrm{CI}$, $0.80-0.97 ; p=0.01)$ in the IVT group and $0.82(95 \%$ CI, $0.66-0.98 ; p=0.047$ ) for patients in the IVTMT group who achieved substantial reperfusion. The NNT for 1 patient achieving functional independence through IVTMT increased with pretreatment lesional volume: from 10 with a lesional volume of approximately $80 \mathrm{ml}$ to 15 with $135 \mathrm{ml}$ and 20 with $160 \mathrm{ml}$ (Fig 5).

In the pretreatment lesional volume $\leq 70 \mathrm{~mL}$ subgroup $(n=245)$, additional MT increased the proportion of patients achieving functional independence by $10.5 \%$ (95\% CI $[-2.7 ; 23.7] ; p=0.13)$, and by $1.9 \%(95 \%$ CI $[-23.2 ; 27.0] ; p=0.99)$ for pretreatment lesional volume

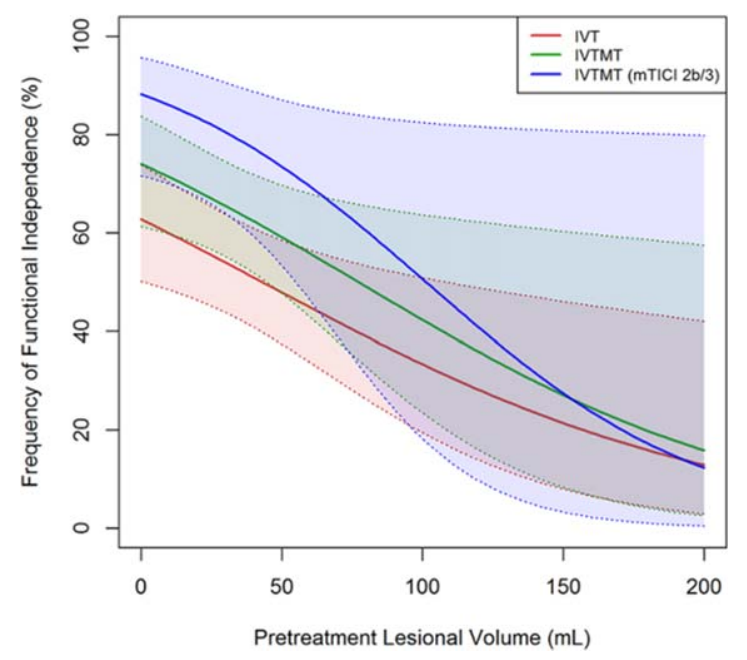

FIGURE 4: Probability of functional independence according to pretreatment lesional volume, by treatment group. Curves were obtained from binomial logistic regression of functional independence (defined by modified Rankin Score $0-2$ ) at 3 months on pretreatment lesional volume as a continuous variable, adjusted for age, baseline NIHSS score, occlusion location of proximal portion of middle cerebral artery, and blood glucose level. The solid lines showed the model results from the logistic regression analysis, with $95 \% \mathrm{Cls}$ shown in the color-corresponding dashed lines. Red lines indicate patients in intravenous thrombolysis (IVT) group; green lines indicate patients in intravenous thrombolysis plus mechanical thrombectomy (IVTMT) group; blue line indicates patients in IVTMT group who achieved substantial reperfusion (modified Thrombolysis in Cerebral Infarction [mTICl] Scale score $2 \mathrm{~b}$ or 3 ). $\mathrm{Cl}=$ confidence interval; NIHSS $=$ National Institutes of Health Stroke Scale. 


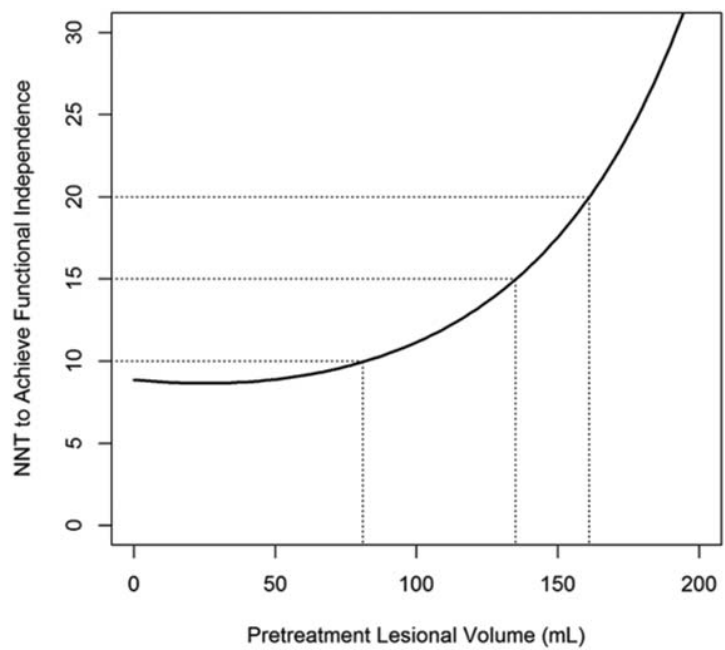

FIGURE 5: Number needed to treat (NNT) for 1 patient achieving functional independence (mRS 0-2) through mechanical thrombectomy according to pretreatment lesional volume. Lesional volume was treated as a continuous variable; the model was adjusted for age, baseline NIHSS score, occlusion location of proximal portion of middle cerebral artery, and blood glucose level. The corresponding lesional volume when NNT was 10, 15, and 20 are shown by dotted lines. $\mathrm{mRS}=$ modified Rankin Scale; NIHSS $=$ National Institutes of Health Stroke Scale.

$>70 \mathrm{ml}(\mathrm{n}=53)$. The increased proportion was $9.2 \%$ (95\% CI $[-3.6 ; 22.0] ; p=0.17)$ for pretreatment lesional volume $\leq 100 \mathrm{ml}(\mathrm{n}=261)$, and 5.0\% (95\% CI [-29.1;

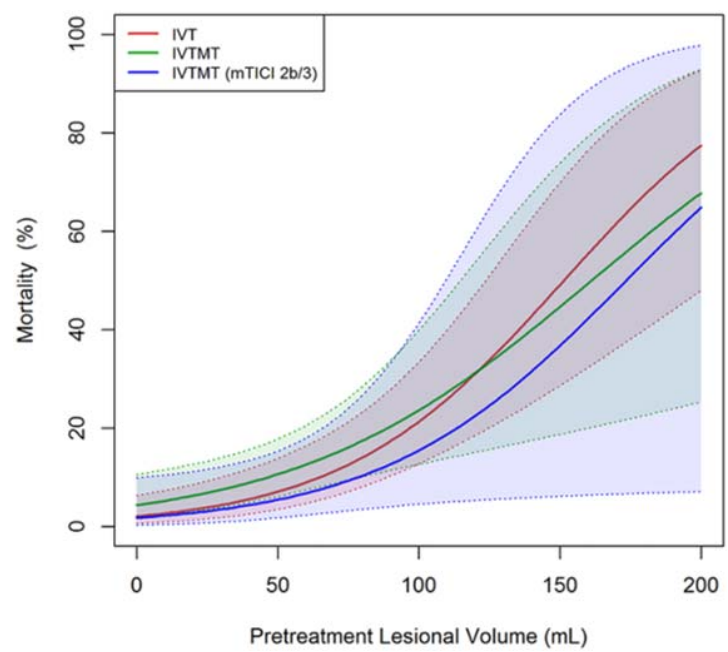

FIGURE 6: Probability of mortality according to pretreatment lesional volume, by treatment group. Curves were obtained from binomial logistic regression of mortality on pretreatment lesional volume as a continuous variable, adjusted for age. The solid lines show the model results from the logistic regression analysis, with $95 \% \mathrm{Cls}$ shown in the color-corresponding dotted lines. Red lines indicate patients in the intravenous thrombolysis (IVT) group; green lines indicate patients in the intravenous thrombolysis plus mechanical thrombectomy (IVTMT) group; blue line indicates patients in the IVTMT group who achieved substantial reperfusion (modified Thrombolysis in Cerebral Infarction [mTICl] Scale score $2 \mathrm{~b}$ or 3 ). $\mathrm{Cl}=$ confidence interval.
39.1]; $p=0.99)$ for pretreatment lesional volume $>100 \mathrm{ml}(\mathrm{n}=37)$. We noted no significant treatment-bydichotomized lesional volume interaction for functional independence (dichotomized by pretreatment lesional volume of $70 \mathrm{ml}$ : OR, 0.99; 95\% CI, 0.19-5.42, $p=0.99$; dichotomized by pretreatment lesional volume of 100ml: OR, 0.98; 95\% CI, 0.16-6.74; $p=0.99$ ). Besides, NNT for 1 patient achieving functional independence through MT compared with thrombolysis alone was similar for patients who had pretreatment lesional volume $\leq 70 \mathrm{ml}$ or $\leq 100 \mathrm{ml}$ (Fig 5).

The mortality rate was $10.8 \%$ (16 of 148 ) in the IVTMT group and $12.0 \%$ (18 of 150) in the IVT group $(p=0.89)$. After adjustment for age, the mortality rate increased with the increase of pretreatment lesional volume (per 10ml: IVTMT group: OR, 1.21; 95\% CI, 1.07-1.38; $p=0.003$; IVT group: OR, 1.29 ; $95 \% \mathrm{CI}$, $1.17-1.45 ; p<0.001$; IVTMT group patients with substantial reperfusion: OR, 1.26; 95\% CI, 1.01-1.60; $p=0.04$; Fig 6). No significant treatment-bydichotomized pretreatment lesional volume interaction was observed for mortality (dichotomized by pretreatment lesional volume of $70 \mathrm{ml}$ : OR, $0.42 ; 95 \% \mathrm{CI}$, $0.08-2.12 ; \quad p=0.30$; dichotomized by pretreatment lesional volume of $100 \mathrm{ml}: \mathrm{OR}, 0.23 ; 95 \% \mathrm{CI}, 0.03-$ $1.39 ; p=0.12)$.

\section{Discussion}

Our study emphasizes the importance of pretreatment lesional volume to predict disability and mortality at 3 months in patients with acute ischemic stroke attributed to proximal intracranial arterial occlusion. Prediction of clinical outcome at the acute stage of ischemic stroke has a number of clinical benefits, ranging from determining optimal therapeutic intervention to informing patients and their family of possible consequences, as well as optimizing patient inclusion criteria for clinical trials.

DWI has been established as the most useful imaging sequence for estimating the ischemic core at the acute stage, because of its high diagnostic sensitivity and specificity, as well as its nonionizing nature. ${ }^{12}$ The results of our THRACE substudy could have implications for clinical routine on account of the semiautomatic volumetric assessment for ischemic lesions in a large population, which was reliable and also easily applicable in a clinical setting. Moreover, patients in the IVTMT group were treated with last-generation MT devices and achieved a high proportion of early reperfusion.

Our study produced four main findings. First, as expected, pretreatment lesional volume was an independent predictor for clinical disability and mortality in our 
cohort, even when reperfusion status was taken into consideration (in the IVTMT group). This result was in accord with previous studies. ${ }^{13-15}$ In a retrospective analysis of the Bernese stroke registry, including magnetic resonance imaging (MRI) triage of stroke patients, Gilgen et $\mathrm{al}^{14}$ observed that the volume of DWI lesions was an independent predictor of unfavorable outcome and survival. The three-dimensional scatterplots with fitted surfaces in our study also illustrate the clear association between pretreatment lesional volume and functional independence at 3 months, according to age and initial stroke severity. Even with young age and mild neurological deficit, which are two well-known predictive factors for a favorable functional outcome, ${ }^{22}$ the probability of achieving functional independence was lower in patients with a large pretreatment lesional volume.

Second, with the increase of pretreatment lesional volume, the NNT to obtain 1 patient achieving 3-month functional independence with IVTMT increased, indicating that the benefit of adding MT to thrombolysis decreased. Also of note, the proportion of patients achieving functional independence in the IVTMT group was always superior to that in the IVT group. Therefore, we can infer the benefit of thrombectomy regardless of pretreatment lesional volume.

Third, previous studies have suggested that patients with a pretreatment lesional volume superior to $70 \mathrm{ml}$ present a poorer outcome, ${ }^{15,16}$ and $100 \mathrm{ml}$ has also been proposed as a potential threshold for patient selection. ${ }^{21}$ After dichotomizing patients by lesional volume of 70 and $100 \mathrm{ml}$, we observed a trend of greater benefit of MT in the subgroups with the smaller volume. However, the nonsignificant treatment-bydichotomized lesional volume interaction for functional independence and mortality suggests that excluding patients based on a simple volume threshold would be unadvisable. It is worth noting that although patients with a large pretreatment lesional volume represented a small proportion of our cohort (53 patients with pretreatment lesional volume $>70 \mathrm{ml}$ ), our results were robust because of the absence of exclusion criteria based on pretreatment lesional volume. Our result is similar to that of a subgroup analysis of the MR CLEAN trial, which found that patients with relatively large estimated pretreatment lesional volume (evaluated by ASPECTS) should be treated, but they included only 30 patients with CT ASPECTS $<5 .{ }^{23}$

Fourth, we confirmed the importance of substantial reperfusion for a favorable functional outcome, as previously reported elsewhere. ${ }^{13,14}$ Gilgen et $\mathrm{al}^{14}$ reported that a favorable outcome was achieved in every third patient with DWI lesions $>70 \mathrm{ml}$ after successful reperfusion, but in only every 12 th patient after poor or failed reperfusion. Olivot et $\mathrm{al}^{13}$ also proved that complete recanalization was associated with a more favorable outcome; however, patients were mainly treated with thrombolysis. In our study, substantial reperfusion significantly increased the proportion of patients achieving functional independence. However, reperfusion status was only investigated in a per-protocol analysis of the IVTMT group, because of the absence of this information for patients in the IVT group and for patients in the IVTMT group who did not receive thrombectomy, because they were not monitored with angiography.

Our study has several limitations. First, a relatively smaller treatment effect of MT than in other randomized clinical trials was observed in the THRACE study, likely attributed to the shorter randomization delay and longer time to groin puncture. We only selected a part of the patients, which might explain the nonsignificant effect of MT on functional independence in our cohort. Moreover, the small number of patients with a large pretreatment lesional volume in our cohort may have resulted in a misestimate of the treatment-by-dichotomized lesional volume interaction. Despite this limitation, our results were robust on account of the absence of exclusion criteria based on pretreatment lesional volume. Second, we selected predefined lesional volume thresholds (70 and $100 \mathrm{ml})$ to test treatment-by-volume interaction according to previous studies; the question of a cohort-specific threshold should be investigated. Third, standardized follow-up data at 24 hours are not available in THRACE. This is because imaging modality in THRACE study was determined according to the local routine at each participating center. A majority of the patients only received follow-up CT examinations at 24 hours instead of MRI. In the end, we only analyzed patients who had pretreatment DWI in the THRACE study. However, CT remains the routine first-line modality in acute stroke evaluation, because it is faster and is easier to access in most of the stroke centers. A further substudy of the THRACE trial is planned to elucidate the influence of the two imaging modalities on clinical outcome.

In conclusion, the results of this subgroup analysis of the THRACE study confirmed the independent value of pretreatment lesional volume in predicting functional outcome in ischemic stroke patients post-MT. The clinical benefit of adding MT to thrombolysis decreased as the pretreatment lesional volume increased. However, excluding patients from treatment according to simple pretreatment lesional volume thresholds would appear unadvisable. 


\section{Acknowledgment}

The THRACE study was funded by the French Ministry for Health as part of its 2009 STIC program for the support of costly innovations (grant number 2009 A0075354). Yu Xie is sponsored by the China Scholarship Council.

We thank Farhat Benbelkacem and Raphaël Petazzoni (Olea Medical) for their help in image postprocessing.

\section{Author Contributions}

Y.X., C.O., F.G., and S.B. contributed to the conception and design of the study. Acquisition and analysis of data were performed by Y.X., C.O., F.G., V.G., H.R., S.S., G.H., and S.B. Y.X., C.O., F.G., B.G., J.F., and S.B. drafted text and/or prepared figures.

\section{Potential Conflicts of Interest}

Nothing to report.

\section{References}

1. Berkhemer OA, Fransen PS, Beumer D, et al. A randomized trial of intraarterial treatment for acute ischemic stroke. N Engl J Med 2015;372:11-20.

2. Saver JL, Goyal M, Bonafe A, et al. Stent-retriever thrombectomy after intravenous t-PA vs. t-PA alone in stroke. $\mathrm{N}$ Engl J Med 2015;372:2285-2295.

3. Goyal M, Demchuk AM, Menon BK, et al. Randomized assessment of rapid endovascular treatment of ischemic stroke. $\mathrm{N}$ Engl J Med 2015;372:1019-1030

4. Campbell BC, Mitchell PJ, Kleinig TJ, et al. Endovascular therapy for ischemic stroke with perfusion-imaging selection. N Engl J Med 2015;372:1009-1018.

5. Jovin TG, Chamorro A, Cobo E, et al. Thrombectomy within 8 hours after symptom onset in ischemic stroke. N Engl J Med 2015;372:2296-2306.

6. Bracard S, Ducrocq X, Mas JL, et al. Mechanical thrombectomy after intravenous alteplase versus alteplase alone after stroke (THRACE): a randomised controlled trial. Lancet Neurol 2016;15: 1138-1147.

7. Muir KW, Ford GA, Messow CM, et al. Endovascular therapy for acute ischaemic stroke: the Pragmatic Ischaemic Stroke Thrombectomy Evaluation (PISTE) randomised, controlled trial. J Neurol Neurosurg Psychiatry 2017;88:38-44.

8. Goyal M, Menon BK, van Zwam WH, et al. Endovascular thrombectomy after large-vessel ischaemic stroke: a meta-analysis of individual patient data from five randomised trials. Lancet 2016; 387:1723-1731.
9. Bucker A, Boers AM, Bot JC, et al. Associations of ischemic lesion volume with functional outcome in patients with acute ischemic stroke. Stroke 2017;48:1233-1240.

10. Al-Ajlan FS, Goyal M, Demchuk AM, et al. Intra-arterial therapy and post-treatment infarct volumes: insights from the ESCAPE Randomized Controlled Trial. Stroke 2016;47:777-781.

11. Albers GW, Goyal M, Jahan R, et al. Relationships between imaging assessments and outcomes in solitaire with the intention for thrombectomy as primary endovascular treatment for acute ischemic stroke. Stroke 2015;46:2786-2794.

12. Edlow JA. Evidence-based guideline: the role of diffusion and perfusion MRI for the diagnosis of acute ischemic stroke: report of the Therapeutics and Technology Subcommittee of the American Academy of Neurology. Neurology 2011;76:177-185.

13. Olivot JM, Mosimann PJ, Labreuche J, et al. Impact of diffusionweighted imaging lesion volume on the success of endovascular reperfusion therapy. Stroke J Cereb Circ 2013;44:2205-2211.

14. Gilgen MD, Klimek D, Liesirova KT, et al. Younger stroke patients with large pretreatment diffusion-weighted imaging lesions may benefit from endovascular treatment. Stroke 2015;46:2510-2516.

15. Yoo AJ, Verduzco LA, Schaefer PW, et al. MRI-based selection for intra-arterial stroke therapy: value of pretreatment diffusionweighted imaging lesion volume in selecting patients with acute stroke who will benefit from early recanalization. Stroke J Cereb Circ 2009;40:2046-2054

16. Šaňák D, Nosál' V, Horák $D$, et al. Impact of diffusion-weighted MRI-measured initial cerebral infarction volume on clinical outcome in acute stroke patients with middle cerebral artery occlusion treated by thrombolysis. Neuroradiology 2006;48:632-639.

17. Straka M, Albers GW, Bammer R. Real-time diffusion-perfusion mismatch analysis in acute stroke. J Magn Reson Imaging 2010; 32:1024-1037.

18. Lansberg MG, Straka M, Kemp S, et al. MRI profile and response to endovascular reperfusion after stroke (DEFUSE 2): a prospective cohort study. Lancet Neurol 2012;11:860-867.

19. Nael K, Knitter JR, Jahan R, et al. Multiparametric magnetic resonance imaging for prediction of parenchymal hemorrhage in acute ischemic stroke after reperfusion therapy. Stroke 2017;48:664670.

20. Cook RJ, Sackett DL. The number needed to treat: a clinically useful measure of treatment effect. BMJ 1995;310:452-454.

21. Schaefer PW, Pulli B, Copen WA, et al. Combining MRI with NIHSS thresholds to predict outcome in acute ischemic stroke: value for patient selection. AJNR Am J Neuroradiol 2015;36:259264.

22. Saposnik G, Guzik AK, Reeves M, et al. Stroke Prognostication using Age and NIH Stroke Scale: SPAN-100. Neurology 2013;80: 21-28.

23. Yoo AJ, Berkhemer OA, Fransen PSS, et al. Effect of baseline Alberta Stroke Program Early CT Score on safety and efficacy of intra-arterial treatment: a subgroup analysis of a randomised phase 3 trial (MR CLEAN). Lancet Neurol 2016;15: 685-694. 